\title{
MOLECULAR DEPLETIONS IN CLOUD CORES
}

An Observational Look at the Gas Phase Depletions

LEE G. MUNDY

Astronomy Dept., Univ. of Maryland

College Park, MD 20742, USA

AND

JOSEPH P. MCMULLIN

SMTO, Steward Observatory

University of Arizona, Tucson, AZ 85721, USA

\begin{abstract}
The condensation of gas-phase molecules onto grain surfaces in cold molecular clouds is widely expected, and the presence of the resultant icy mantles well established, but quantitative study of the gas-phase depletions has not proved easy. This paper reviews the methods for determining depletions and the associated problems. Further observations are critical to testing our expectations for depletions and for the chemical evolution of forming stellar systems.
\end{abstract}

\section{Introduction}

The expectation that molecules should condense onto the surfaces of grains in the dense, cold regions of molecular clouds has a long history (Watson \& Salpeter 1972; Greenberg \& Hong 1974; Allen \& Robinson 1977; Iglesias 1977; Wootten et al. 1980). The motivation for expecting these depletions arises from the consideration that gas molecules will hit and stick to grain surfaces if the grain temperature is significantly below the desorption temperature. In the simple case where a molecule sticks to the grain surface for a fraction $\alpha$ of the collisions, the timescale for molecules to deplete from the gas phase is given by

$$
\tau_{\text {depl }}=\frac{2 \times 10^{9}}{\alpha n_{H_{2}}} \text { years }
$$

where $\mathrm{n}_{\mathrm{H}_{2}}$ is the gas density of $\mathrm{H}_{2}$. For gas densities typical of dense molecular cores, $10^{5} \mathrm{~cm}^{-3}$ to several $10^{6} \mathrm{~cm}^{-3}$, and $\alpha=1$, the depletion timescale 
is 1,000 to a few $10^{4}$ years. Since molecular clouds live for a few times $10^{7}$ years and dense cores live for at least $10^{6}$ years (c.f. Blitz 1993), depletions should be widespread.

The fact that dark clouds are easily seen in molecular tracers indicates that molecular depletions cannot be as complete as suggested above. Either $\alpha$ must be much less than unity or other processes besides thermal evaporation remove molecules from grain surfaces. A number of desorption processes have been suggested including spot heating by UV photons, cosmic rays, and $\mathrm{H}_{2}$ formation, chemically driven explosions, and shock heating by grain collisions or sputtering (c.f. Léger et al. 1985; Schutte \& Greenberg 1991). The efficiencies of each of these processes, and their applicability in different environments, is a matter of debate but it is clear that the observed depletions result from competition between condensation and desorption.

This paper concentrates on quantitative expectations for, and observational determinations of, gas-phase depletions. This is only half of the story since the molecules depleted from the gas are incorporated into icy mantles which can be studied at infrared wavelengths but, perhaps surprisingly, gas-phase depletions are not well understood, despite more than two decades of observations.

\section{Models of molecular depletions}

A number of papers over the past 20 years have modeled the time evolution of molecular abundances in the presence of depletion and desorption processes (Allen \& Robinson 1977; Iglesias 1977; Boland \& de Jong 1982; Millar \& Nejad 1985; Brown et al. 1988; Charnley et al. 1988; Chièze et al. 1991; Rawlings et al. 1992; Bergin et al. 1995). These papers broadly define quantitative expectations for what depletions should be present in molecular clouds. To illustrate these expectations, we chose three of these works for brief review.

Rawlings et al. (1992) calculate the chemical evolution of the collapsing envelope of a protostar including depletion onto grains. They followed the chemistry and dynamics of the envelope over roughly a free-fall time. Their Figure 1 shows the characteristic behavior of a number of molecular species. Most abundances remain constant at the initial values for $10^{6}$ years then drop precipitously near $10^{7}$ years. The drop is caused by rapidly increasing depletions as the density of the collapsing material increases. The strength of this work is its combination of chemistry and dynamics which permits the calculation of line profiles and the identification of molecular ions such as $\mathrm{HCO}^{+}$and $\mathrm{N}_{2} \mathrm{H}^{+}$as important species which are resistant to depletion. Its weakness is the lack of desorption mechanisms so that by $10^{7}$ years all 
molecules are condensed into ices.

The second paper, Charnley et al. (1988), explores one of many possible scenarios for dynamically cycling material on-and-off grain surfaces (for other scenarios see Boland \& de Jong 1982; Chièze et al. 1991; Xie et al. 1995). Charnley et al. look at models in which interclump gas collapses to form clumps; the clumps remain static for a while, then are heated and dispersed by young stellar winds. They look at this as a recurring cycle with molecules depleting onto grains during the collapse and static phases and returning to the gas during the dispersal phase. The resulting abundances show significant time variations. The amount of depletion depends on how long the gas remains in the cold clump phase.

The third paper, Bergin et al. (1995), looks at time dependent chemistry in the presence of depletion and desorption. They find cosmic ray induced desorption is dominant deep within molecular clouds. Models with only thermal evaporation show strong depletions on the timescale of a few times $10^{6}$ years, in agreement with Rawlings et al.; with cosmic ray and photodesorption, the gas-phase abundances of most species remain significant for periods of greater than $10^{7}$ years, although abundances for most species peak around $10^{5}$ years. In particular, they find that many of the commonly observed tracer molecules show depletions of over an order of magnitude for a gas density of $10^{5} \mathrm{~cm}^{-3}$.

In summary, very weak or no depletions are expected throughout the bulk of molecular clouds because the density is modest (200 to $3000 \mathrm{~cm}^{-3}$ ) and the visual extinction moderate (1 to 5 magnitudes). Modest to large depletions should occur in quiescent cores where the density is $10^{4}$ to $10^{6} \mathrm{~cm}^{-3}$ and the heating due to radiation and shocks is weak. Large depletions are associated with the densest cores, $n=10^{6}$ to $10^{8} \mathrm{~cm}^{-3}$, as long as vigorous star formation is not occurring. The radiative heating and shock activity associated with massive star formation, and even winds from low-mass star formation, can return condensates to the gas phase and end depletions. These are the expectations; what is seen?

\section{Observational determinations of depletions}

To date, observational determinations of gas-phase molecular abundances and molecular depletions in molecular clouds have mainly used radio wavelength, rotational transitions of common molecular species (CO, CS, HCN, $\mathrm{H}_{2} \mathrm{CO}$ ) or their less abundant isotopes. Three basic approaches have been utilized in deriving depletions: molecular $-\mathrm{H}_{2}$ column density and molecular mass comparisons which yield absolute depletions, and relative column density comparisons which yield relative depletions.

In the first approach, the column density of a molecule is calculated 
from the observed integrated line intensity using,

$$
N(\text { molecule }) \propto Q\left(T_{e x}\right) e^{\frac{E_{u}}{k T_{e x}}} \int T_{o b s} d V
$$

where $\mathrm{Q}$ is the partition function, $T_{e x}$ is the excitation temperature, $E_{u}$ is the energy of the upper state of the transition, $T_{o b s}$ is the observed line temperature, and $\int \mathrm{dV}$ is the integral of the line emission over velocity. Thus, the observed emission from a single line can be converted into a molecular column density if $T_{e x}$ is known. The description of the molecular excitation by a single $T_{e x}$ is a simplification which is not always justified. In the general case, $T_{e x}$ is derived from observations of two or more lines of the molecule. The $\mathrm{H}_{2}$ column density in molecular cores is usually determined from the dust emission,

$$
N\left(\mathrm{H}_{2}\right) \propto \frac{S_{\nu}}{\kappa_{\nu}}\left(e^{\frac{h \nu}{k T_{d u s t}}}-1\right)
$$

where $\kappa_{\nu}$ is the mass opacity coefficient at the frequency of observation, $S_{\nu}$ is the observed flux, and $T_{d u s t}$ is the dust temperature. $\kappa_{\nu}$ is only known to within a factor of 2 to 4 and estimation of $T_{d u s t}$ can be problematic due to the presence of multiple temperature components along the line of sight. The absolute fractional abundance is then

$$
X(\text { molecule })=\frac{N(\text { molecule })}{N\left(\mathrm{H}_{2}\right)} \propto T_{d u s t} T_{e x}^{0 \text { to } 1}
$$

which is dependent on both the molecular excitation temperature and the dust temperature. The $T_{e x}$ dependence in the above equation is a function of details of the molecular excitation. Hence, a factor of two error in the temperature determinations can result in a factor of four error in the abundance.

The second approach uses the mass determined from spatially integrating the molecular emission over the core and the Virial mass of the core.

$$
M_{\mathrm{mol}}=\int_{\text {clump }} N(\text { molecule }) d \Omega / X(\text { molecule })
$$

where $N$ (molecule) is the column density of the molecule. The Virial mass is given by $M_{v i r}=3 R \sigma^{2} / G$, where $R$ is the radius of the core and $\sigma$ is the velocity dispersion. Assuming that the two masses are equal,

$$
X(\text { molecule })=\frac{M_{\text {mol }}}{M_{\text {vir }}}
$$

This approach requires the core to be spatially resolved so that $R$ can be measured. Only a core averaged abundance can be derived in this way and 
the core is assumed to be gravitationally bound, which may not be true (c.f. Blitz 1993).

A third approach is to derive relative abundances rather than absolute abundances. This can be done as the ratio of the column densities of two species, with $\mathrm{CO}$ usually serving as the reference species.

$$
\frac{X(\text { molecule })}{X(\mathrm{CO})}=\frac{N(\text { molecule })}{N(\mathrm{CO})}
$$

Since column density is easily derived from observations and the ratio of molecular column densities should generally have a weak dependence on $T_{e x}$, relative abundances are usually reliable. However, it is only a relative abundance; inferred relative depletions are subject to uncertainties caused by changes in the abundance of the reference species.

All of these methods for determining abundances and gas-phase depletions have significant uncertainties associated with them. In fact, few molecular line or continuum studies of molecular clouds or cloud cores are able to draw significant conclusions regarding gas-phase depletions. In the next two sub-sections we review the depletion evidence for two example regions: a massive cloud core where a young stellar core is forming, and a small core where a low-mass system is forming.

\subsection{THE NGC 2024 MOLECULAR RIDGE}

The NGC 2024 Molecular Ridge provides an interesting case history showing the difficulty establishing depletions in complex regions. Mezger et al. (1988) began the story with observations of the $\lambda=1300$ and $350 \mu \mathrm{m}$ dust emission from a several hundred solar mass ridge of material associated with the NGC 2024 embedded, young cluster; the dust emission does not follow the molecular emission distribution as traced by $\mathrm{CS}$ and $\mathrm{C}^{18} \mathrm{O}$. From modeling of the emission, Mezger et al. inferred the presence of cold condensations which are severely depleted of molecules. Moore et al. (1989) and Schultz et al. (1991) called these depletions into question based on molecular line observations which showed gas temperatures of $30-50 \mathrm{~K}$. These temperatures are much higher than those found by Mezger et al. and, if true, weaken the argument for depletions.

Companion papers, Mezger et al. (1992) and Mauersberger et al. (1992), revisited the depletion issue with new dust continuum and molecular line observations which emphasize the poor correlation between the two tracers. The main condensations, which are obvious in the dust emission, are barely visible in isotopic $\mathrm{CS}$ and $\mathrm{C}^{18} \mathrm{O}$ emission. They again find low dust temperatures, and inferred gas temperatures, which lead to the derivation of molecular depletions. The poor spatial correlation between the dust continuum and molecular line emission is explored with higher spatial resolution 
by Wilson et al. (1995) with interferometer observations. They found compact continuum emission associated with three of the condensations identified by Mezger et al. $(1988,1992)$ but the compact $\mathrm{C}^{18} \mathrm{O}$ emission was generally offset from the continuum emission. Wilson et al. (1995) inferred depletions in $\mathrm{C}^{18} \mathrm{O}$ of factors of 6 to 10 in the three clumps.

But, the story isn't over. Based on interferometer maps of the CS $J=2-1$ line and dust continuum, Chandler \& Carlstrom (1996) argue that all of the observations can be explained without invoking depletions if the gas and dust temperatures in the condensations are $\geq 100 \mathrm{~K}$. Mangum et al. (1996) in this meeting present a poster which argues for gas temperatures of 40 to $80 \mathrm{~K}$ in the condensation, or at least in molecular gas along the ridge. These temperatures are close to those needed for Chandler and Carlstrom's argument. Based on an analysis of the submillimeter dust emission, Visser et al. (1996), in a poster at this meeting, suggest that the dust emissivity changes within the condensations. If true, the estimation of $\mathrm{H}_{2}$ column densities for the clumps becomes problematic and the depletions more uncertain.

\subsection{NGC 1333 IRAS 4: AN EXTREMELY EMBEDDED STELLAR SYSTEM}

A second type of region with evidence for gas-phase depletions is illustrated by the young source IRAS 4 in the NGC 1333 molecular cloud. IRAS 4 is a deeply embedded low-luminosity multiple system which is bright at submillimeter and millimeter wavelengths but invisible at visible and nearinfrared wavelengths (Sandell et al., 1991). The two molecular cores surrounding the forming stars contain between 1 and 8 solar masses of material and have a size scale of $\sim 1000$ AU (Sandell et al. 1991; Blake et al. 1995). Based on comparison of dust continuum and molecular line emission, Blake et al. argue for depletions by factors of $10-20$ in all molecular abundances, including $\mathrm{CO}$, within these cores.

Higher resolution interferometer observations of these cores find even stronger depletions and a striking lack of correspondence between continuum and line emission and between single-dish and interferometer line profiles (McMullin, 1994). In the $\mathrm{C}^{18} \mathrm{O}$ line, the interferometer image shows no emission from the two cores over the bright part of the single-dish line profile. The $\mathrm{C}^{18} \mathrm{O}$ abundance appears depleted by factors of $20-200$ within the individual cores. In the case of IRAS 4, the dust and gas temperatures are well constrained by observations so the major uncertainty in the depletions is due to uncertainty in the dust emissivity. If ices are growing on grain surfaces, and if grains are undergoing coagulation, it is possible that the grain emissivity is changing making the calculation of $N\left(\mathrm{H}_{2}\right)$ problematic. 


\section{Alternative evidence for gas-phase depletions}

Enhancements in the gas-phase abundances of selected molecules due to the evaporation of ices provide secondary evidence for depletions. For example, some deuterium-bearing molecules show strongly enhanced abundances compared to their corresponding non-deuterated isomers in cold molecular clouds. This enhancement is due to the lower energy of the deuterated species; hence the enhancements disappear in warm environments. However, in some regions of recent star formation, deuterated molecules, such as HDO (Plambeck \& Wright, 1987), $\mathrm{CH}_{3} \mathrm{OD}$ (Mauersberger et al., 1988), and DCN (Mangum et al., 1991), show relative abundances comparable to those found in dark clouds. Based on the spatial distribution and kinematics, it is argued that these species were incorporated into icy grain mantles when the cloud was cold and now are returning to the gas phase as star formation heats and evaporates the ices.

Evaporating grain mantles have also been invoked to help understand strong enhancements of methanol associated with some molecular outflows (McMullin et al. 1993; Sandell et al. 1994; Bachiller et al. 1995; Avery \& Chiao 1996). In these outflow sources, the $\mathrm{CH}_{3} \mathrm{OH}$ emission, which

arises from gas with a temperature of $\sim 100 \mathrm{~K}$ and a density of $\sim 10^{6} \mathrm{~cm}^{-3}$, is found to trace portions of the outflow lobe, yet shows nearly ambient cloud velocities. These characteristics suggest that the methanol is being evaporated or sputtered from grain mantles in weak shock interactions between the outflow and ambient cloud (Blake et al. 1995; also see references above). The evaporation of significant icy reservoirs of molecular material is likely to play a central role in explaining many of the chemical enhancements associated with outflow activity and radiative heating (Charnley et al. 1992).

\section{Pitfalls and ponderings}

The two primary objectives in studying gas-phase depletions in molecular clouds are to quantify the depletions in different environments and to characterize the physical processes that control the degree of depletion. We are not yet close to achieving these objectives but the results to date highlight several important points.

First, high resolution observations are essential to study depletions in any region where star formation is active, and this includes most regions with gas densities over $10^{5} \mathrm{~cm}^{-3}$. Second, good measures of the gas and dust temperatures are necessary to derive depletions with accuracies of better than a factor of 4 . The hard part is that dust and gas temperatures must be measured in the same material in which the depletions are occurring. Third, there is growing evidence that dust emissivity changes from clump- 
to-clump or region-to-region in clouds (Wright et al. 1992; Visser et al. 1996). This introduces a significant additional uncertainty in the derivation of depletions unless the dust emissivity can be established for the region under study.

Finally, the easiest quantities to measure are molecular column densities, but these are integrals along the line of sight. Cloud densities, temperatures, and molecular abundances can vary significantly along the line of sight; these variations are difficult to deconvolve unless the source geometry is known or the kinematics particularly favorable. Without such as a deconvolution, un-depleted material along the line of sight limits the measured depletion factors. Since one hopes to measure depletions of factors of tens to hundreds, a small amount of un-depleted material goes a long way in masking severe depletions.

TABLE 1. A Chemical Evolutionary Sequence

\begin{tabular}{ll}
\hline Dynamical Status & Chemical Status \\
\hline Identifiable core & Normal Abundances \\
Contracting core & $\begin{array}{l}\text { Weak depletions at center, } \\
\text { increasing with time } \\
\text { Strong depletions in center, } \\
\text { weak depletions throughout }\end{array}$ \\
Stellar object forms & $\begin{array}{l}\text { Maximum depletions } \\
\text { Shock enhancements begin }\end{array}$ \\
Wind and photon heating & $\begin{array}{l}\text { Shock enhancements and grain } \\
\text { mantle evaporation near star }\end{array}$ \\
& and spreading out with time \\
& Evaporation of mantles throughout \\
Appearance of star & core
\end{tabular}

\section{Onward toward a chemical evolutionary sequence}

From the observational studies and the theoretical modeling to date, it is clear that the molecular morphology of dense regions of molecular clouds is determined by the interplay of depletions, desorptions, and the chemistry driven by evaporation of volatile species. Over the next few years, infrared observations of solid-phase and gas-phase species from ISO and high resolution observations of gas-phase species from millimeter interferometers will enable broader quantitative work on depletions and the identification of desorption processes. As outlined in Table 1, at present, we have a rough expectation for how the chemical evolution of star-forming regions tracks 
the dynamical evolution. More observations are needed to test, refine, and redefine these expectations.

\section{References}

Allen, M., Robinson, G.W. 1977, ApJ 212, 396.

Avery, L.W., Chiao, M. 1996, ApJ 463, 642.

Bachiller, R., Liechti, S., Walmsley, C.M., Colomer, F. 1995, A\&A 295, L51.

Bergin, E.A., Langer, W.D., Goldsmith, P.F. 1995, ApJ 441, 222.

Blake, G.A., Sandell, G., van Dishoeck, E.F., Groesbeck, T.D., Mundy, L.G., Aspin, C. 1995, ApJ 441, 689.

Blitz, L. 1993, in Protostars and Planets III, ed. Levy, E.H. and Lunine, J.I. (University of Arizona Press:Tucson), p.125.

Boland, W., de Jong, T. 1982, ApJ 261, 110.

Brown, P.D., Millar, T.J., Charnley, S.B. 1988, MNRAS 231, 409.

Chandler, C.J., Carlstrom, J.E. 1996, ApJ 466, 338.

Charnley, S,B., Tielens, A.G.G.M., Millar, T.J. 1992, ApJ 399, L71.

Charnley, S.B., Dyson, J.E., Hartquist, T.W., Williams, D.A. 1988, MNRAS 235, 1257.

Chièze, J.P. Pineau des Forêts, G., Herbst, E. 1991, ApJ 373, 110.

Greenberg, J.M., Hong, S. 1974, in Galactic Radio Astronomy, IAU Symposium 60, eds. F.J. Kerr and S.C. Swanson (Reidel: Dordrecht), p.155.

Iglesias, E. 1977, ApJ 218, 697.

Léger, A., Jura, M., Omont, A. 1985, A\&A 144, 147.

Mangum, J.G., Plambeck, R.L., Wootten, A. 1991, ApJ 369, 169.

Mangum, J., Wootten, A., Barsony, M. 1996, in IAU 178 Symposium Abstract book, Molecules in Astrophysics: Probes 8 Processes, eds. D.J. Jansen, M.R. Hogerheijde and E.F. van Dishoeck (Leiden Observatory), p. 85.

Mauersberger, R., Henkel, C., Jacq, T., Walmsley, M. 1988, A\&A 194, L1.

Mauersberger, R., Wilson, T.L., Mezger, P.G., Gaume, R., Johnston, K.J. 1992, A\&A 256,640 .

McMullin, J.P. 1994, PhD Thesis, Univ. of Maryland.

McMullin, J.P., Mundy, L.G., Blake, G.A. 1993, ApJ 405, 599.

Mezger, P.G., Chini, R., Kreysa, E., Wink, J.E., Salter, C.J. 1988, A\&A 191, 44.

Mezger, P.G., Sievers, A.W., Haslam, C.G.T., Kreysa, E., Lemke, R., Mauersberger, R., Wilson, T.L. 1992, A\&A 256, 631.

Millar, T.J., Nejad, L.A.M. 1985, MNRAS 217, 507.

Moore, T.J.T., Chandler, C.J., Gear, W.K., Mountain, C.M. 1989, MNRAS 237, 1p.

Plambeck, R.L., Wright, M.C.H. 1987, ApJ 317, L101.

Rawlings, J.M.C., Hartquist, T.W., Menten, K.M., Williams, D.A. 1992, MNRAS 255, 471.

Sandell, G., Aspin, C., Duncan, W.D., Russell, A.G.G., Robson, E.I. 1991, ApJ 376, L17.

Sandell, G., Knee, L.B.G., Aspin, C., Robson, I.E., Russell, A.P.G. 1994, A\&A 285, L1.

Schultz, A., Gusten, R., Zylka, R., Serabyn, E. 1991, A\&A 246, 570.

Schutte, W.A., Greenberg, J.M. 1991, A\&A 244, 190.

Visser, A., Padman, R., Richer, J. 1996, in IAU 178 Symposium Abstract book, Molecules in Astrophysics: Probes $\&$ Processes, eds. D.J. Jansen, M.R. Hogerheijde and E.F. van Dishoeck (Leiden Observatory), p. 86.

Watson, W.D., Salpeter, E.E. 1972, ApJ 175, 659.

Wilson, T.L., Mehringer, D.M., Dickel, H.R. 1995, A\&A 303, 840.

Wright, M.C.H., Sandell, G., Wilner, D.J., Plambeck, R.L. 1992, ApJ 393, 225.

Wootten, A., Snell, R., Evans, N.J.II 1980, ApJ 240, 532.

Xie, T., Allen, M., Langer, W.D. 1995, ApJ 440, 674. 


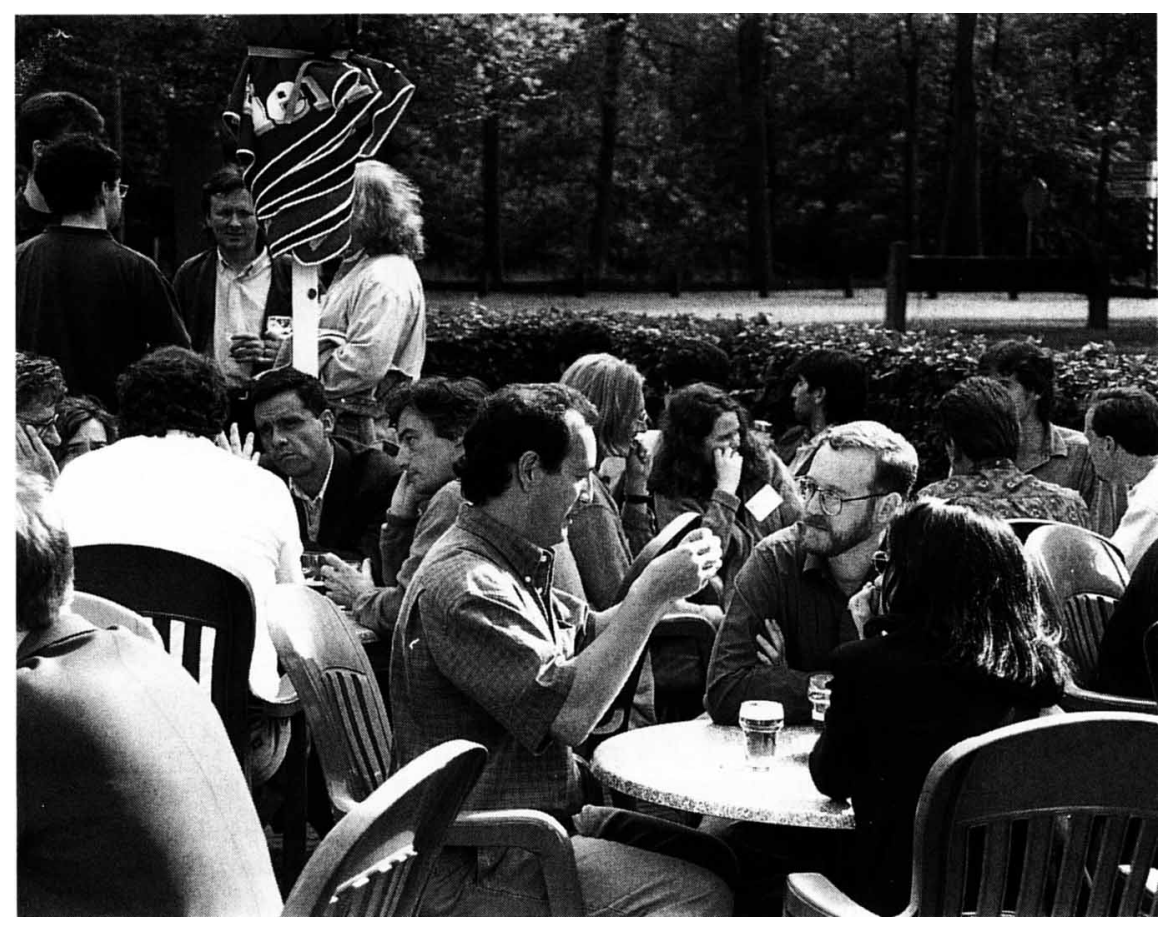

